\title{
Elevage de bovins Somba et gestion de son écologie parasitaire gastro-intestinal au Bénin
}

\author{
Tiropa Francis CHABI CHINA ${ }^{1 *}$, Armand Bienvenu GBANGBOCHE ${ }^{1,2}$, \\ Sabbas ATTINDEHOU², Sanni-Yô DOKO ALLOU ${ }^{2}$, Sahidou SALIFOU ${ }^{3}$, \\ Louis Joseph PANGUI ${ }^{4}$ et François Adébayo ABIOLA ${ }^{3}$
}

\author{
${ }^{I}$ Faculté des Sciences Agronomiques - Université d'Abomey-Calavi, 01 BP 526 Cotonou, Bénin. \\ ${ }^{2}$ Ecole de Gestion et d'Exploitation des Systèmes d'Elevage, Université d'Agriculture de Kétou, \\ BP 43 Kétou, Bénin. \\ ${ }^{3}$ Ecole Polytechnique d'Abomey Calavi. 01 BP 5009 Université d'Abomey Calavi. Cotonou. Bénin. \\ ${ }^{4}$ Ecole Inter-Etats des Sciences et Médecine Vétérinaires de Dakar. B.P. 5077 Dakar Sénégal. \\ *Auteur correspondant,Email: tiropaacc@yahoo.fr ; Tél: (+229) 97604862
}

\section{RESUME}

Le bovin Somba est confronté à des contraintes liées à son milieu naturel dont principalement le parasitisme. Dans le but d'avoir une meilleure connaissance des conditions d'élevages de ce bovin en milieu réel, les performances obtenues, et la gestion des facteurs parasitaires d'influence, une revue bibliographique des travaux scientifiques réalisés dans ce sens a été effectué. Il en ressort que le bovin Somba évolue dans de mauvaises conditions d'élevage qui limitent fortement ses performances productives. Les parasites favorisés par un climat chaud et les mauvaises conditions d'élevage sévissent gravement dans les troupeaux. La chimiothérapie, moyen de lutte classique connait aujourd'hui des limites qui ont amené à explorer d'autres possibilités telles que l'ethnomédecine vétérinaire dont l'utilisation reste encore informelle et sans référentiel médical. Ainsi, l'amélioration des conditions d'élevage et un meilleur suivi sanitaire prenant en compte la valorisation de l'ethnopharmacie sont indispensables pour des élevages de bovins Somba plus productifs et plus viables.

(C) 2016 International Formulae Group. All rights reserved.

Mots clés : Taurins Somba, trypanotolérant, ethnomédecine, performances zootechniques, parasitisme.

\section{Somba cattle breeding and management of its gastrointestinal parasitic ecology in Benin}

\begin{abstract}
Somba cattle breed is faced with constraints related to the natural environment which mainly parasitism. In order to have a better knowledge of farming conditions of the cattle in the real environment, the resulting performance, and management of parasitic influencing factors, a bibliographic review of scientific work in this direction has been made. It shows that bovine Somba evolves in poor farming conditions which severely limit its productive performance. Parasites favored by a warm climate and poor farming conditions
\end{abstract}


seriously prevalent in herds. Chemotherapy, conventional means of control now knows the limitations that led to explore other possibilities like ethnomedecine whose use is still informal and without medical repository. Thus, improvement of breeding conditions and better health monitoring taking into account the valuation of ethnomdecine are essential for farms more productive and more sustainable cattle Somba.

(C) 2016 International Formulae Group. All rights reserved.

Keywords: Somba cattle, trypanotolerant, ethnomedecine, zootechnic performances, parasitism.

\section{INTRODUCTION}

La race bovine Somba est l'une des races autochtones de l'Afrique de l'Ouest. De ce fait, elle a acquis une grande rusticité dans son milieu naturel en plus du fort potentiel de production qu'elle pourrait exprimer dans de bonnes conditions d'élevage. C'est une race $\mathrm{du}$ groupe des bovins trypanotolérants d'Afrique de l'Ouest sans bosse (Bos taurus) appelés taurins comme l'indique la Figure 1 qui présente la répartition des principales races bovines en Afrique Occidentale et Centrale. Elle a été introduite en Afrique via l'Egypte à partir des populations de taurins originaires de l'Asie du Sud-Ouest (Bos taurus nomadicus), qui appartiendraient à deux grands groupes : les taurins à longues cornes (Bos indicus primigenus), déjà présents en Egypte 5000 ans avant Jésus-Christ, et les taurins à courtes cornes (Bos taurus brachyceros) arrivés environ en 2000 ans avant Jésus-Christ (Moazami-Goudarzi et al., 2001). Les taurins à longues cornes ou West African Long Horn, ont effectué une première migration Nord-Sud, passant par le Soudan, puis une seconde migration Est-Ouest, passant par le Tchad pour se retrouver en Afrique Occidentale. Les courtes cornes, à travers un mouvement migratoire Nord-Sud se sont implantées sur les côtes atlantiques. Encore appelés West African Short Horn, les taurins à courtes cornes, comportent deux groupes (les races naines à courtes cornes qui regroupent les types de race de Lagune et les races typiques à courte corne comme la race Somba dont le berceau est la région située à cheval entre le Nord-Ouest du Bénin et le Nord-Est du Togo. Les bovins somba représentés sur la Figure 2 et les lagunaires sont deux races qui présentent de nette différence génétique, alors qu'ils passaient pour être très proches, voire identiques (Moazami-Goudarzi et al., 2001), les robes étant comparables de pie-noires et noires suivies de robes fauves, mouchetées ou truitées (Adanléhoussi, 2003). Au Bénin, l'effectif des bovins somba en 1987 qui était de 216.000 têtes a considérablement chuté au seuil de 25.000 têtes en 1995 , puis à 17.000 têtes à nos jours (Shaw et al., 1987 ; Dossa, 2000 ; PAMRAD, 2006) et se trouve dispersé dans une grande partie du département de l'Atacora (notamment dans les communes de Boukoumbé, Natitingou, Toucountouna, Matéri, Cobli, Tanguiéta). Au Togo le bovin somba est encore dénommé bovin Mango (une ville située dans la région des savanes), et se retrouve également dans les cantons de Nadoba, Koutougou, et la préfecture de la Kéran (Adanléhoussi, 2003). La qualité principale de cette race est son adaptation à un environnement humide (zone guinéenne et soudano-guinéenne) défavorable à l'élevage en général des mammifères domestiques, à cause de la présence de la trypanosomose, la dermatophylose, les parasitoses diverses et les maladies transmises par les tiques. $\mathrm{La}$ trypanotolérance est donc un atout très important pour la promotion des bovins dans les milieux infestés de glossine (MoazamiGoudarzi et al., 2001). Techniquement, il n'est pas possible à l'heure actuelle d'éradiquer les glossines de certains groupes, mais assurer le contrôle pour abaisser la densité est l'alternative plausible. L'avantage d'utiliser le bétail trypanotolérant est de mise et depuis 1995 , et les auteurs recommandent la conservation et la valorisation de ce groupe génétique de bovins (Hall et al., 1995). Cependant, la race Somba fait l'objet de peu d'attention (Dossa, 2000 ; Adanléhoussi, 
2003) et le défi majeur est d'éviter son extinction ou son absorption par le cheptel Zébu compte tenu de la diminution drastique des effectifs enregistré depuis quelques décennies. Dans la perspective d'actions postérieures de tentative de sauvegarde et de promotion de la race Somba, la présente étude est donc réalisée pour avoir une meilleure connaissance de cette race, ses performances productives et reproductives, le milieu dans lequel il évolue, les contraintes liées à ce milieu, et la gestion des principaux facteurs d'influence notamment les parasites gastro-intestinaux.

\section{CARACTERISTIQUES DES BOVINS SOMBA}

Les bovins Somba sont élevés aussi bien au Bénin qu'au Togo respectivement par les ethnies Tamari et Temberma qui détiennent de longues pratiques sur ces animaux qui évoluent tous dans un système de type sédentaire (Adanlehoussi et al., 2003; Chabi China et al., 2013). On reconnait deux types de troupeaux de Somba, selon l'importance et l'appartenance des animaux : le troupeau familial et le troupeau confié. Dans le troupeau familial, la taille du cheptel est de 3 à 10 bêtes, les animaux sont conduits et surveillés au pâturage par un ou plusieurs membres de la famille et le soir sont parqués dans les tatas. Le "troupeau confié", quant à lui est sous la conduite d'un bouvier peulh contre une rémunération annuelle forfaitaire et du droit de traire les vaches (PAMRAD, 2006 ; Chabi China et al., 2013). Ce sont des troupeaux composites de 40 à 100 bêtes appartenant à plusieurs (5 à 30) propriétaires. Ces derniers visitent de façon plus irrégulière les animaux et interviennent que pour des décisions importantes telles que les ventes et achats de tous genres.

En termes de performances de développement corporel et de croissance, la hauteur au garrot $(\mathrm{HG})$ du bovin somba varie de $0,90 \mathrm{~cm}$ à 1 mètre. Très proche du bovin lagunaire, il est difficile de faire le distinguo d'un veau somba et d'un lagunaire (Domingo, 1976). D'après Joshi et al. (1957) la HG chez l'adulte est de $114 \mathrm{~cm}$ (vache), $115 \mathrm{~cm}$ (boeuf) et $116 \mathrm{~cm}$ (taureau) avec un périmètre thoracique (PT) correspondant respectivement à 136, 137 et $139 \mathrm{~cm}$ (Tableau 1). A l'exception de la lagunaire qui a pratiquement les mêmes caractéristiques morphologiques que la Somba, les autres races taurines c'est à dire le Borgou, le Baoulé, le Kapiski, le N'dama présentent un format plus relevé (Tableau 2). La possibilité d'utiliser le périmètre thoracique (PT) pour estimer le poids des Somba a été mise en exergue par Adanléhoussi et al. (2003) en se référant sur le choix des coefficients de détermination $\left(\mathrm{R}^{2}\right)$ variant entre 0,90 à 0,98 (Tableau 3 ). Ce qui lui permet de déterminer un poids à l'âge adulte situé entre 165 et $209 \mathrm{~kg}$. L'effet du dimorphysme sexuel est également observé avec la race Somba car les mâles ont tendance à être plus lourds que les femelles de la naissance jusqu'à l'âge adulte (Hall et al., 1995). D'une bonne aptitude bouchère, le rendement à l'abattage du bovin Somba est de 43-52\% selon Hardouin (1989) et Hall et al. (1995). Bien qu'inappropriée à la traction animale, quelques cas contraires ont été reportés au Togo.

Par rapport aux performances de reproduction, Joshi et al. (1957) ont rapporté l'âge au premier vêlage (APV) de 3,5 ans et l'intervalle entre vêlage (IV) de 14 mois, bien que Hardouin et al. (1989) signalent 3 ans (APV) et 11 mois (IV). Selon Hall et al. (1995), l'APV dans les conditions villageoises ou traditionnelles varie entre 36 et 48 mois et l'IV de 12 à 14 mois. D'autres auteurs dans les mêmes conditions ont rapporté la durée de 48 mois pour l'APV, de 22 mois pour l'IV, suivi d'un taux de fécondité de 40\% (SedogboHouenou, 1993). Ce dernier avoisine $60 \%$ en station pourrait aller à $69 \%$ (Adanléhoussi et al., 2003). Le mâle atteint sa maturité sexuelle à 2 ans $1 / 2$ et demeure actif jusqu'à $12-14$ ans (Avégan, 1984). La production de lait montre des tendances variables, mais sans grandes différences, l'écart entre auteurs ne dépasse guère 0,5 litres : entre 1,5 à 2 litres $/ \mathrm{j}$ (Domingo, 1976) et 1 à $1,5 \mathrm{l} /$ jour pendant 180 à 200 jours de lactation (Sedogbo-Houenou, 
1993). Cette production est très faible. Toutefois une simple amélioration de la conduite et de l'alimentation des races produisant généralement $0,5 \mathrm{l} / \mathrm{j}$ de lait, conduise à produire plus de 2 litres $/ \mathrm{j}$. Généralement la race Somba exprime une bonne fertilité et peut atteindre 5 à 7 rangs de lactation (Avegan, 1984).

\section{RISQUES PARASITAIRES DANS L'ECOSYSTEME DES BOVINS SOMBA}

Depuis des décennies, les parasitoses sont classées au rang des principales contraintes sanitaires pour le cheptel bovin béninois. Les pathogènes les plus incriminés sont les coccidies, les strongles gastrointestinaux, les piroplasmes et les trypanosomes. Cette tendance reste d'actualité et dans le septentrion notamment, région de prédilection pour le cheptel bovin au Bénin (Youssao et al., 2013), la trypanosomose et la piroplasmose sont les parasitoses les plus préoccupantes. Si la trypanotolérance de la race Somba est avérée, elle reste fortement vulnérable au danger que constituent les hémoparasites des genres Babesia, Theileria et Anaplasma. En effet, les bovins Somba et même toutes les autres races bovines évoluant dans le Nord Bénin sont continuellement et intensément confrontés aux tiques vectrices de ces pathogènes. Farougou et al. (2007) ont signalé que dix (10) espèces de tiques sévissent sur les bovins du Nord-Bénin avec une prédominance des espèces Boophilus geigeyi (32,68\%), Amblyomma variegatum (23,5\%), Rhipicephalus senegalensis $(11,22 \%)$, Boophilus annulatus $(7,72 \%)$ et Hyalomma marginatum $(6,39 \%)$ (Tableau 4). Le spectre helminthique quant à lui, se constitue notamment des trématodes des genres Fasciola, Paramphistomum et Dicrocoelium et des strongles digestifs, Haemonchus en premier (Tableaux 5 et 6). Assogba et Youssao (2001) ont rapporté une forte prévalence des trématodoses dues à Fasciola gigantica, Paramphistomum sp. et à Dicrocoelium horpes. De nombreux auteurs ont travaillé ces dernières années sur la lutte ethnovétérinaire contre l'haemonchose quoique la prévalence de cette strongylose et son importance chez les bovins restent non élucidées. Il est tout de même établi grâce aux données fournies par Attindéhou et al. (2012) que le strongle hématophage, Haemonchus contortus (Figures 3) sévit bien dans l'aire de répartition du bovin Somba. Il en est de même pour d'autres strongyloses communes aux ruminants en général.

La race bovine somba qui est l'une des races autochtones de l'Afrique de l'Ouest a acquis une grande rusticité dans son milieu naturel en plus du fort potentiel de production tout en cohabitant avec de nombreux parasites internes et externes. Dans de bonnes conditions d'élevage, notamment par une bonne gestion des risques parasitaires, le bovin Somba devrait sans doute être plus productif.

\section{LA GESTION DES RISQUES PARASITAIRES}

Outre les recherches en cours sur les possibilités de modifier le patrimoine génétique des animaux afin de les rendre plus résistants aux agressions de l'environnement, en particulier celles qui mettent en cause les parasites ou agents infectieux, et le tarissement des sources de contamination, deux moyens de luttes contre les risques parasitaires sont dans les pratiques déployés sur le terrain: la lutte chimique et l'ethnomédecine vétérinaire.

\section{LA LUTTE CHIMIQUE}

Depuis de nombreuses années, l'emploi de molécules anthelminthiques de synthèse est le moyen le plus utilisé contre ces parasites dans les élevages de bovins Somba bénéficiant de suivi d'agents vétérinaires. Trois familles de molécules anthelminthiques (Benzimidazoles, Imidazothiazole et Lactones macrocycliques) (Tableau 7) sont réputées efficaces et préconisées par les agents vétérinaires pour lutter contre les strongles gastro-intestinaux des ruminants (Lacroux 2006 ; Besier, 2007). Mais de plus en plus cette chimiothérapie est controversée. En effet, outre les restrictions d'emploi pour les 
femelles en gestation et les femelles allaitantes, certaines interrogations concernant l'utilisation quasi-exclusive de la chimiothérapie anthelminthique sont instamment formulées ici et là (Lumaret et Errouissi, 2002 ; Lacroux, 2006 ; Bishop et Morris, 2007 ; Brunet, 2008) face au phénomène de développement de résistance par de nombreux parasites. En effet, d'après Waller (2006), une population de parasites résistantes à la chimiothérapie est une population ayant génétiquement acquis la capacité de résister à des concentrations d'anti-parasitaires habituellement létales pour les individus de cette espèce. Ainsi, le déterminisme de la résistance qui est génétique, peut être considéré comme un phénomène pré-adaptatif. $\mathrm{La}$ pression environnementale (comme l'utilisation des anthelminthiques) sélectionne certaines mutations dans une population de parasites. Le succès reproductif de ces individus résistants est plus important que celui des individus sensibles. Comme le temps de génération est court chez les strongles, la proportion d'individus résistants augmente rapidement dans la population (Prichard, 2001).

\section{L'ETHNOMEDECINE VETERINAIRE}

Compte tenu des problèmes ci-dessus évoqués, certains éleveurs recourent aux méthodes endogènes de traitement. Ces méthodes basées sur les plantes, proviennent généralement de connaissances ancestrales transmises de génération en génération (Assogbadjo et al., 2009; Déléké - Koko et al., 2011; Dibong et al., 2011; Dossou et al., 2012; Fah et al., 2013). Le mode d'utilisation de ces méthodes est très variable d'un éleveur à un autre, et leur efficacité effective sur les affections supposées traitées reste à établir scientifiquement avant qu'elles ne soient une alternative crédible à la chimiothérapie chez les bovins. Depuis quelques années de nombreuses études sont initiées dans ce sens afin de prouver l'efficacité et le bien-fondé de l'utilisation des méthodes endogènes à base de plantes comme alternative à la médecine vétérinaire moderne. C'est ainsi que Athanasiadou et al. $(2000 ; 2001)$ avaient déjà évoqué la possibilité d'action antiparasitaires directe de certaines légumineuses fourragères et plus particulièrement des plantes riches en tanins. Depuis lors, de nombreux travaux ont été menés dans le but de tester les éventuelles aptitudes anthelminthiques de plusieurs plantes notamment en région tropicale. Au Bénin, Hounzangbé-Adoté (2004), et Olounlandé et al. (2011), ont prouvé les propriétés anthelminthiques de quatre plantes tropicales (Zanthoxylum zanthoxyloides, Newbouldia laevis, Morinda lucida, Carica papaya) in vitro et in vivo sur les nématodes gastro-intestinaux chez les ruminants. Bien d'autres plantes tropicales telles que Azadirachta indica (Costa et al., 2006), Leucaena leucocephalla (Adémola et al., 2006), Acacia nilotica (Kahiya et al., 2003), Trifolium repens (Bernes, G. et al., 2000), Adansonia digitata (De Caluwé et al., 2009; Nguta et al., 2010), Bridelia ferruginia (Adetutu et al., 2011 ; Fabiyi et al., 2012 ; Lagnika et al., 2012), Carica papaya (Zunjar et al., 2011 ; Milind et Gurditta, 2012 ; Varisha et al., 2013) etc. ont également été testées avec succès sur les parasites gastrointestinaux des ruminants. 
Tableau 1: Mensurations et pesées des bovins de race Somba.

\begin{tabular}{|c|c|c|c|c|c|c|c|c|c|}
\hline Tranches d'âges & Effectif & HG (cm) & PT (cm) & LSI (cm) & LH (cm) & IH (cm) & LT (cm) & IT (cm) & Poids (kg) \\
\hline Naissance & & & & & & & & & 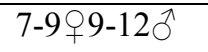 \\
\hline $0-15$ jours & 29 & $51,57 \pm 3,55$ & $55,28 \pm 2,62$ & $51,00 \pm 3,35$ & $16,4 \pm 0,54$ & $11,00 \pm 0,81$ & $18,42 \pm 1,39$ & $10,57 \pm 2,29$ & $14,16 \pm, 04$ \\
\hline $15-30$ jours & 20 & $57,00 \pm 1,29$ & $58,00 \pm 1,41$ & $55,15 \pm 1,41$ & $18,01 \pm 0,5$ & $12,00 \pm 0,5$ & $19,5 \pm 0,57$ & $11,50 \pm 0,57$ & $17,25 \pm 1,41$ \\
\hline $1-3$ mois & 39 & $60,28 \pm 3,81$ & $65,10 \pm 5,31$ & $60,28 \pm 5,87$ & $19,00 \pm 1,63$ & $13,42 \pm 0,78$ & $22,00 \pm 2,00$ & $12,16 \pm 0,75$ & $22,25 \pm, 50$ \\
\hline 3-6 mois & 80 & $63,00 \pm 3,20$ & $72,75 \pm 1,25$ & $65,40 \pm 4,65$ & $21,00 \pm 0,50$ & $15,00 \pm 0,50$ & $24,00 \pm 1,25$ & $13,00 \pm 0,57$ & $31,25 \pm, 06$ \\
\hline $6-12$ mois & 204 & $70,42 \pm 6,47$ & $83,71 \pm 7,25$ & $74,42 \pm 8,24$ & $24,57 \pm 2,30$ & $18,28 \pm 1,60$ & $28,57 \pm 3,20$ & $14,00 \pm 1,15$ & $46,18 \pm 12,40$ \\
\hline $1-2$ ans & 208 & $82,60 \pm 9,12$ & $100,00 \pm 14,01$ & $89,20 \pm 12,2$ & $28,00 \pm 3,46$ & $23,20 \pm 4,26$ & $33,40 \pm 4,21$ & $16,00 \pm 1,73$ & $81,30 \pm 15,88$ \\
\hline $2-3$ ans & 216 & $88,12 \pm 4,24$ & $116,43 \pm 7,08$ & $101,06 \pm 6,69$ & $34,20 \pm 1,82$ & $27,06 \pm 2,23$ & $37,31 \pm 1,88$ & $17,43 \pm 1,15$ & $119,00 \pm 9,67$ \\
\hline $3-4$ ans & 135 & $93,38 \pm 1,63$ & $125,92 \pm 1,82$ & $108,00 \pm 5,47$ & $35,00 \pm 0,81$ & $30,00 \pm 1,41$ & $39,50 \pm 0,57$ & $18,25 \pm 0,50$ & $149,38 \pm 5,47$ \\
\hline 4-5 ans & 73 & $97,06 \pm 2,06$ & $128,08 \pm 1,91$ & $113,00 \pm 1,41$ & $36,00 \pm 0,57$ & $31,00 \pm 0,50$ & $41,00 \pm 0,50$ & $19,39 \pm 0,80$ & $158,52 \pm 10,80$ \\
\hline $5-6$ ans & 78 & $96,65 \pm 0,95$ & $129,75 \pm 2,21$ & $114,00 \pm 1,25$ & $36,00 \pm 0,01$ & $31,68 \pm 0,06$ & $41,00 \pm 0,50$ & $19,00 \pm 0,03$ & $165,33 \pm 9,97$ \\
\hline $6-10$ ans & 303 & $96,60 \pm 3,84$ & $131,18 \pm 6,72$ & $116,63 \pm 5,22$ & $37,36 \pm 1,56$ & $32,09 \pm 2,02$ & $41,54 \pm 1,36$ & $19,36 \pm 1,20$ & $172,18 \pm 13,26$ \\
\hline$>10$ ans & 232 & $96,66 \pm 3,80$ & $132,44 \pm 5,45$ & $115,61 \pm 7,17$ & $37,44 \pm 1,14$ & $33,38 \pm 1,28$ & $41,11 \pm 1,96$ & $20,01 \pm 0,94$ & $172,15 \pm 15,51$ \\
\hline
\end{tabular}

HG : Hauteur au Garrot PT : Périmètre Thoracique ; LSI : Longueur Scapulo-Ischiale LH : Longueur de la Hanche ; IH : largeur de la Hanche LT : Longueur de la Tête IT : largeur de la Tête Sources :

Domingo et al.(1976); Grell et al.(1982); Vegan (1984); Adanléhoussi et al. (2003). 
Tableau 2: Mensurations, Performances productives et reproductives de la race Somba comparées aux autres races taurines africaines.

\begin{tabular}{|c|c|c|c|c|c|c|}
\hline Paramètres & $\begin{array}{l}\text { Somba } \\
\text { milieu } \\
\text { Paysan }\end{array}$ & $\begin{array}{l}\text { Somba } \\
\text { (station) }\end{array}$ & Lagunaire & Kapsiki & Baoulé & Borgou \\
\hline \multicolumn{7}{|l|}{ Format } \\
\hline $\mathrm{HG}(\mathrm{cm})$ & 96,6 & & 95 & 106-109 & $99-105$ & 110 \\
\hline PT $(\mathrm{cm})$ & 132,44 & & 136,3 & $140-142$ & 134-141 & 145,3 \\
\hline $\begin{array}{l}\text { Poids moyen adulte } \\
(\mathrm{kg})\end{array}$ & $165-187$ & $200-209$ & 209 & 206 & $166-213$ & \\
\hline \multicolumn{7}{|l|}{ Production } \\
\hline Poids de naissance $(\mathrm{kg})$ & 12 & 21,4 & & & $14-15,9$ & \\
\hline & & 20,6 & & & 13 & \\
\hline GMQ (0-8 mois) (g/j) & 93,15 & $341-208$ & & & $154-170$ & 250 \\
\hline $\begin{array}{l}\text { Productivité de veau } \\
\text { pour } 100 \mathrm{~kg} \text { vache }(\mathrm{kg})\end{array}$ & 15,92 & 29,73 & & & 17,2 & \\
\hline \multicolumn{7}{|l|}{ Reproduction } \\
\hline $\begin{array}{l}\text { Age au } 1^{\text {er }} \text { vêlage } \\
\text { (mois) }\end{array}$ & $\begin{array}{l}36-48 \\
65,50\end{array}$ & $36-39$ & $\begin{array}{l}36-42 \\
42-48\end{array}$ & 48 & 26 & 42 \\
\hline $\begin{array}{l}\text { Intervalle entre } \\
\text { vêlages (mois) }\end{array}$ & $\begin{array}{l}12-14 \\
18,49\end{array}$ & 15,3 & 1824 & & 1419 & $15-17$ \\
\hline Taux de vêlage (\%) & 60,9 & $\begin{array}{l}60 \\
65,5-69\end{array}$ & 43 & & 61 & $60-65$ \\
\hline Taux de mortalité (\%) & 3,37 & 5,3 & & & & 65,4 \\
\hline
\end{tabular}

Tableau 3: description des équations barymétriques chez les bovins de race Somba.

\begin{tabular}{llc}
\hline Tranches d'âge & Equations & Coeff. de détermination \\
\hline $\mathbf{0 - 1}$ an & $\mathrm{P}=1,13 \mathrm{PT}-49,26$ & 0,98 \\
$\mathbf{1 - 4}$ ans & $\mathrm{P}=2,68 \mathrm{PT}-188,31$ & 0,98 \\
$>\mathbf{4}$ ans & $\mathrm{P}=3,5 \mathrm{PT}-288,25$ & 0,90 \\
Tous âges confondus & $\mathrm{P}=139.10^{-6}(\mathrm{PT})^{2,88}$ & 0,98 \\
\hline
\end{tabular}


Tableau 4: Abondance des tiques dans quelques localités du nord Bénin.

\begin{tabular}{|c|c|c|c|c|c|c|c|c|c|c|c|c|c|c|}
\hline \multirow[t]{3}{*}{ Espèces de tiques } & \multicolumn{6}{|c|}{ Septembre } & \multicolumn{6}{|c|}{ Octobre } & \multirow{2}{*}{\multicolumn{2}{|c|}{$\begin{array}{l}\text { Total } \\
(\mathrm{n}=240)\end{array}$}} \\
\hline & \multicolumn{2}{|c|}{$\begin{array}{l}\text { Borgou-Alibori } \\
(\mathrm{n}=60)\end{array}$} & \multicolumn{2}{|c|}{$\begin{array}{l}\text { Atacora-Donga } \\
(\mathrm{n}=60)\end{array}$} & \multicolumn{2}{|c|}{$\begin{array}{l}\text { Nord-Benin } \\
(\mathrm{n}=120)\end{array}$} & \multicolumn{2}{|c|}{$\begin{array}{l}\text { Borgou- } \\
\text { Alibori } \\
(\mathrm{n}=60)\end{array}$} & \multicolumn{2}{|c|}{$\begin{array}{l}\text { Atacora-Donga } \\
(\mathrm{n}=60)\end{array}$} & \multicolumn{2}{|c|}{$\begin{array}{l}\text { Nord-Benin } \\
(\mathrm{n}=120)\end{array}$} & & \\
\hline & $\mathrm{Mm}$ & $\mathrm{A}(\%)$ & $\mathrm{Mm}$ & $\mathrm{A}(\%)$ & $\mathrm{Mm}$ & $\mathrm{A}(\%)$ & $\mathrm{Mm}$ & $\mathrm{A}(\%)$ & $\mathrm{Mm}$ & $\mathrm{A}(\%)$ & $\mathrm{Mm}$ & $\mathrm{A}(\%)$ & $\mathrm{Mm}$ & A $(\%)$ \\
\hline $\begin{array}{l}\text { Amblyomma } \\
\text { variegatum }\end{array}$ & 140 & 19,7 & 72 & 42,1 & 212 & 24,0 & 61 & 12 & 82,5 & 48,5 & 143 & 21,6 & 355 & 23,05 \\
\hline $\begin{array}{l}\text { Boophilus } \\
\text { annulatus }\end{array}$ & 57 & 8,04 & 6,5 & 3,80 & 63,5 & 7,21 & 42 & 8,5 & 13,5 & 7,94 & 55,5 & 8,38 & 119 & 7,72 \\
\hline Boophilus geigyi & 228 & 32,1 & 61 & 35,6 & 289 & 32,8 & 169 & 34 & 46 & 27,0 & 215 & 32,4 & 504 & 32,68 \\
\hline $\begin{array}{l}\text { Hyalomma } \\
\text { impressum }\end{array}$ & 23 & 3,24 & 8,5 & 4,97 & 31,5 & 3,58 & 15 & 3,0 & 5,5 & 3,23 & 20,5 & 3,10 & 52 & 3,37 \\
\hline $\begin{array}{l}\text { Hyalomma } \\
\text { marginatum }\end{array}$ & 48 & 6,77 & 7 & 4,09 & 55 & 6,25 & 36 & 7,3 & 7,5 & 4,41 & 43,5 & 6,57 & 98,5 & 6,39 \\
\hline $\begin{array}{l}\text { Hyalomma } \\
\text { truncatum }\end{array}$ & 41 & 5,78 & 9 & 5,26 & 50 & 5,68 & 31 & 6,3 & 11 & 6,47 & 42 & 6,34 & 92 & 5,97 \\
\hline $\begin{array}{l}\text { Hyalomma } \\
\text { nitidium }\end{array}$ & 27 & 3,81 & 0 & 0 & 27 & 3,07 & 20 & 4,0 & 0 & 0 & 20 & 3,02 & 47 & 3,05 \\
\hline $\begin{array}{l}\text { Rhipicephalus } \\
\text { muhsamae }\end{array}$ & 48 & 6,77 & 0 & 0 & 48 & 5,45 & 39 & 7,9 & 1 & 0,59 & 40 & 6,04 & 88 & 5,71 \\
\hline $\begin{array}{l}\text { Rhipicephalus } \\
\text { senegalensis }\end{array}$ & 90 & 12,6 & 7 & 4,09 & 97 & 11,0 & 73 & 14 & 3 & 1,76 & 76 & 11,4 & 173 & 11,22 \\
\hline $\begin{array}{l}\text { Rhipicephalus } \\
\text { sulcatus }\end{array}$ & 7 & 0,99 & 0 & 0 & 7 & 0,79 & 6 & 1,2 & 0 & 0 & 6 & 0,91 & 13 & 0,84 \\
\hline Total & 709 & & 171 & & 880 & & 492 & & 170 & & 662 & & 1542 & \\
\hline
\end{tabular}

$\mathbf{n}$ : nombre de prélèvements ; Mm : moyenne mensuelle ; $\mathbf{A}$ : abondance

Source: Farougou et al., 2007. 
Tableau 5: Caractéristiques des principaux genres de parasites internes chez les bovins.

\begin{tabular}{|c|c|c|c|c|}
\hline Parasites & Description & $\begin{array}{l}\text { Organe } \\
\text { infecté }\end{array}$ & Cycle de vie & Symptômes \\
\hline Haemonchus & $\begin{array}{l}\mathrm{M}: 10-20 \mathrm{~mm} \text { rouges } \\
\mathrm{F}: 18-30 \mathrm{~mm} \text { rouges et } \\
\text { blanches }\end{array}$ & Caillette & $\begin{array}{l}\text { SI: 4-6 jours } \\
\text { PP: } 3 \text { semaines }\end{array}$ & $\begin{array}{l}\text { anémie, enflures molles sous la } \\
\text { mâchoire et l'abdomen, affaiblissement, } \\
\text { pas de gain de poids }\end{array}$ \\
\hline Ostertagia & $\begin{array}{l}\text { M:6-9 mm, bruns } \\
\text { F:8-12 mm }\end{array}$ & Caillette & $\begin{array}{l}\text { SI: } 4-6 \text { jours } \\
\text { PP: } 3 \text { semaines }\end{array}$ & $\begin{array}{l}\text { même que pour Haemonchus et aussi } \\
\text { inappétence, diarrhée }\end{array}$ \\
\hline $\begin{array}{l}\text { Tricho- } \\
\text { strongylus }\end{array}$ & $\begin{array}{l}\text { M:4-5,5 mm } \\
\text { F:5-7 mm } \\
\text { brun pâle }\end{array}$ & $\begin{array}{l}\text { Caillette, } \\
\text { intestin } \\
\text { grêle }\end{array}$ & $\begin{array}{l}\text { SI: } 3-4 \text { jours } \\
\text { PP: } 2-3 \text { semaines }\end{array}$ & $\begin{array}{l}\text { même que pour Haemonchus et aussi } \\
\text { diarrhée et perte de poids }\end{array}$ \\
\hline Cooperia & $\begin{array}{l}\text { rouges } \\
\mathrm{M}: 5-7 \mathrm{~mm} \\
\mathrm{~F}: 6-9 \mathrm{~mm}\end{array}$ & $\begin{array}{l}\text { Intestin } \\
\text { grêle }\end{array}$ & $\begin{array}{l}\text { SI: } 5-6 \text { jours } \\
\text { PP: } 15-20 \text { jours }\end{array}$ & mêmes que pour Haemonchus \\
\hline Bunostomum & $10-30 \mathrm{~mm}$ & $\begin{array}{l}\text { Intestin } \\
\text { grêle }\end{array}$ & $\begin{array}{l}\text { SI: } \\
\text { PP: } 30-56 \text { jours }\end{array}$ & $\begin{array}{l}\text { Oedème, anémie, perte de poids, } \\
\text { diarrhée }\end{array}$ \\
\hline $\begin{array}{l}\text { Strongyloides } \\
\text { (jeunes animaux) }\end{array}$ & $4-6 \mathrm{~mm}$ & $\begin{array}{l}\text { Petit } \\
\text { intestin }\end{array}$ & $\begin{array}{l}\text { SI: } 1-2 \text { jours } \\
\text { PP: } 8-14 \text { jours }\end{array}$ & anorexie, entérite, diarrhée \\
\hline Chabertia & $\begin{array}{l}\text { M:13-14 mm } \\
F: 17-20 \mathrm{~mm}\end{array}$ & $\begin{array}{l}\text { Gros } \\
\text { intestin }\end{array}$ & $\begin{array}{l}\text { SI: } 5-6 \text { jours } \\
\text { PP: } 42 \text { jours }\end{array}$ & anémie, diarrhée avec sang \\
\hline Oesophago-stomum & $\begin{array}{l}\text { M:12-17 mm } \\
F: 15-22 \mathrm{~mm}\end{array}$ & $\begin{array}{l}\text { Gros } \\
\text { intestin }\end{array}$ & $\begin{array}{l}\text { SI: } 6-7 \text { jours } \\
\text { PP: } 41-45 \text { jours }\end{array}$ & diarrhée vert foncé, œdème \\
\hline $\begin{array}{l}\text { Proto- } \\
\text { strongylus }\end{array}$ & $\begin{array}{l}\text { M:16-28 mm } \\
F: 25-35 \mathrm{~mm}\end{array}$ & Poumons & $\begin{array}{l}\text { SI: } 12-14 \text { jours } \\
\text { PP: } 30-37 \text { jours }\end{array}$ & pneumonie \\
\hline Dictyocaulus & $\begin{array}{l}\text { M:30-80 mm } \\
F: 50-100 \mathrm{~mm}\end{array}$ & Poumons & $\begin{array}{l}\text { SI: } 6-7 \text { jours } \\
\text { PP: } 3-4 \text { semaines }\end{array}$ & $\begin{array}{l}\text { écoulement nasal collant, respiration } \\
\text { difficile, toux }\end{array}$ \\
\hline
\end{tabular}


Tableau 6: Principales espèces de trichostrongles gastro-intestinaux des ruminants et leur localisation dans le tube digestif.

\begin{tabular}{|c|c|c|c|}
\hline Sous-famille & Espèces & Localisation chez l'hôte & Hôtes \\
\hline \multirow[t]{3}{*}{ Haemonchinae } & Haemonchus contortus & Abomasum & Ovins, Caprins \\
\hline & Haemonchus placei & Abomasum & Bovins \\
\hline & Haemonchus longistipes & Abomasum & Dromadaires \\
\hline \multirow[t]{2}{*}{ Trichostrongylinae } & Trychostrongylus colubriformis & Intestin grêle & Ovins, Caprins \\
\hline & Trychostrongylus axei & Abomasum & Caprins, Ovins, Bovins \\
\hline \multirow[t]{2}{*}{ Ostertagiinae } & Teladorsagia circumcincta & Abomasum & Ovins, Caprins \\
\hline & Ostertagia ostertagi & Abomasum & Bovins \\
\hline \multirow[t]{2}{*}{ Cooperlinae } & Cooperia curicei & Intestin grêle & Caprins, Ovins, Bovins \\
\hline & Cooperia oncophora & Intestin grêle & Ovins, Bovins \\
\hline
\end{tabular}

Tableau 7: Molécules anthelminthiques couramment utilisées.

\begin{tabular}{|c|c|c|c|c|}
\hline Famille & Molécule & Nom déposé & Posologie & Mode d'action \\
\hline \multirow[t]{4}{*}{ Benzimidazoles } & Oxfendazole & $\begin{array}{l}\text { Oxfenil } \\
\text { Synanthic }\end{array}$ & $5 \mathrm{mg} / \mathrm{kg}$ Voie orale & \multirow{4}{*}{$\begin{array}{l}\text { Détruisent les nématodes en inhibant la formation de la } \beta \\
\text { tubuline en micro tubule et provoquent ainsi le blocage de la } \\
\text { multiplication cellulaire et la dégénérescence des cellules } \\
\text { intestinales du parasite. } \\
\text { Détruisent et perturbent le développement des œufs. }\end{array}$} \\
\hline & Fenbendazole & Panacur & $5 \mathrm{mg} / \mathrm{kg}$ Voie orale & \\
\hline & Fébantel & Rintal & $5 \mathrm{mg} / \mathrm{kg}$ Voie orale & \\
\hline & Albendazole & Valbazen & $3,8 \mathrm{mg} / \mathrm{kg}$ Voie orale & \\
\hline Imidazothiazole & Lévamisole & $\begin{array}{l}\text { Lévamisole } \\
\text { Némisol }\end{array}$ & $7.5 \mathrm{mg} / \mathrm{kg}$ Voie orale & $\begin{array}{l}\text { Paralysent les vers par fixation sur les récepteurs post- } \\
\text { synaptiques des nématodes. Inhibent les récepteurs } \\
\text { d'acétylcholine provoquant la paralysie des larves et des vers } \\
\text { adultes }\end{array}$ \\
\hline \multirow{4}{*}{$\begin{array}{c}\text { Lactones } \\
\text { macrocycliques }\end{array}$} & Ivermectine & Ivomec & $0.2 \mathrm{mg} / \mathrm{kg}$ Sous cutanée & \multirow{4}{*}{$\begin{array}{l}\text { Agissent par fixation sur les récepteurs au glutamate des } \\
\text { canaux chlore des cellules des parasites. Cela provoque la } \\
\text { paralysie du pharynx par afflux d'ions } \mathrm{Cl} \text {. }\end{array}$} \\
\hline & & Oramec & $0.2 \mathrm{mg} / \mathrm{kg}$ Voie orale & \\
\hline & Doramectine & Dectomax & $\begin{array}{l}0.2 \mathrm{mg} / \mathrm{kg} \text { Intra-musculaire ou } \\
\text { Sous cutanée }\end{array}$ & \\
\hline & Moxidectine & Cydectine & $\begin{array}{l}0.2 \mathrm{mg} / \mathrm{kg} \text { Voie orale ou Sous } \\
\text { cutanée }\end{array}$ & \\
\hline
\end{tabular}

Source : Lacroux, 2006. 


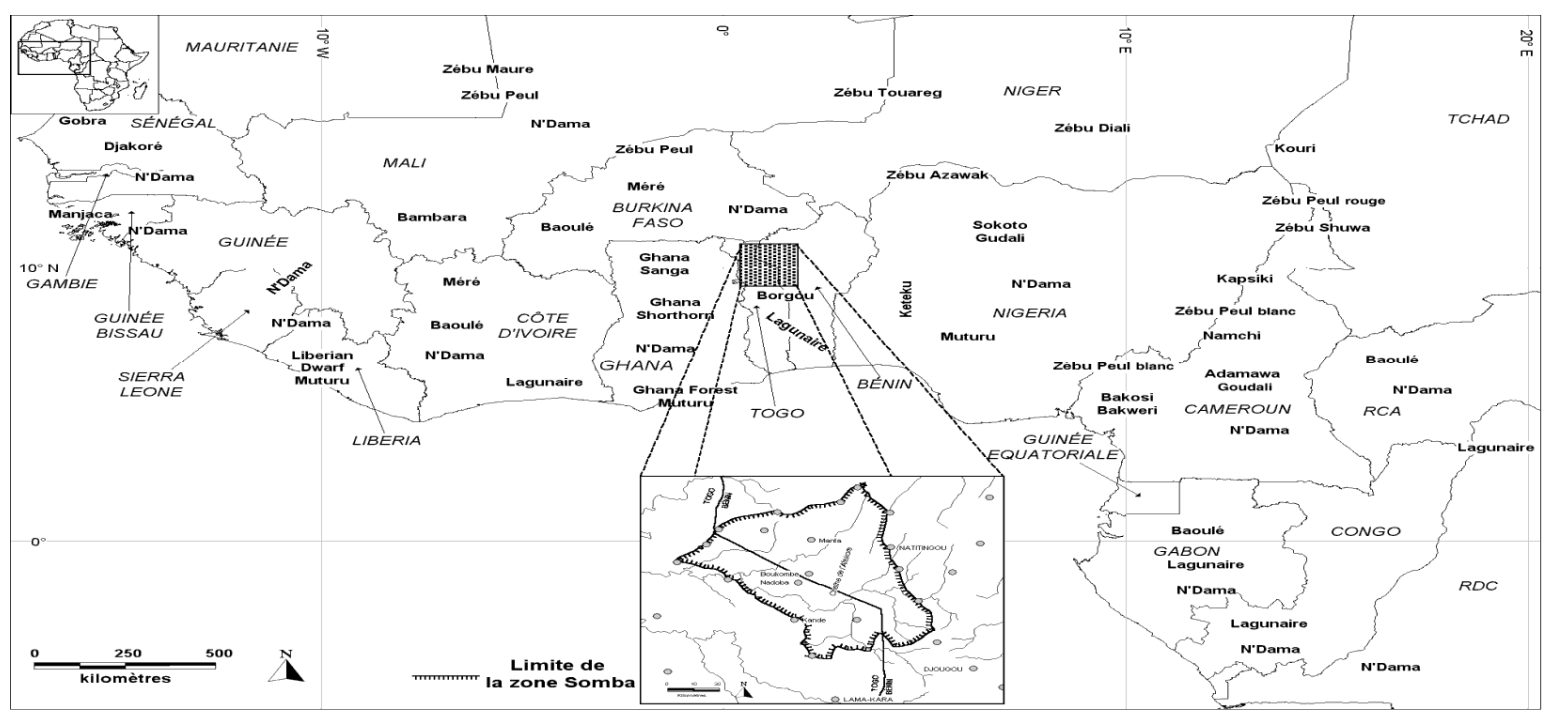

Figure 1: Répartition des principales races de bovins en Afrique occidentale et centrale (MoazamiGoudarzi et al., 2001).

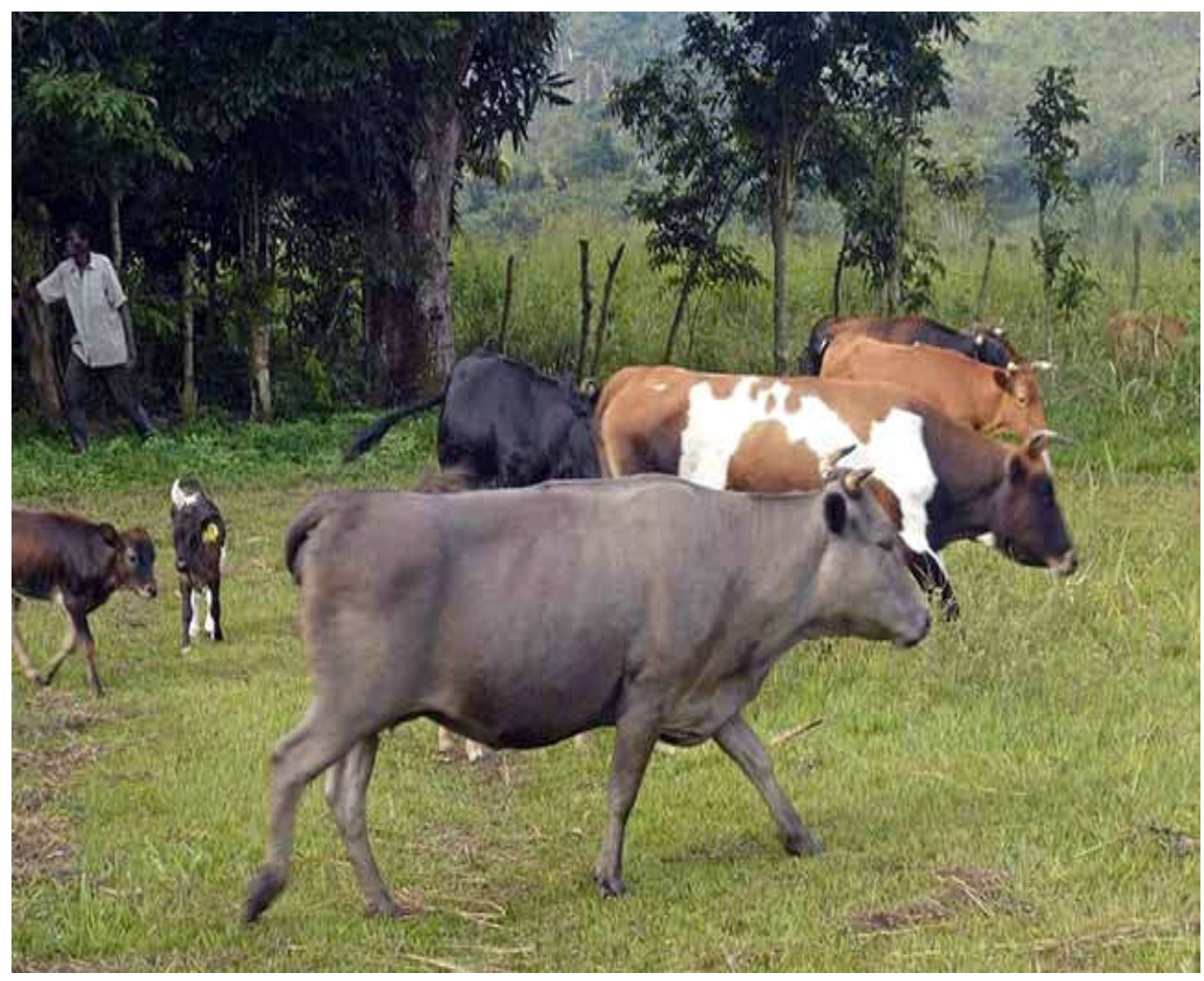

Figure 2: Le bovin Somba (PAMRAD, 2006). 


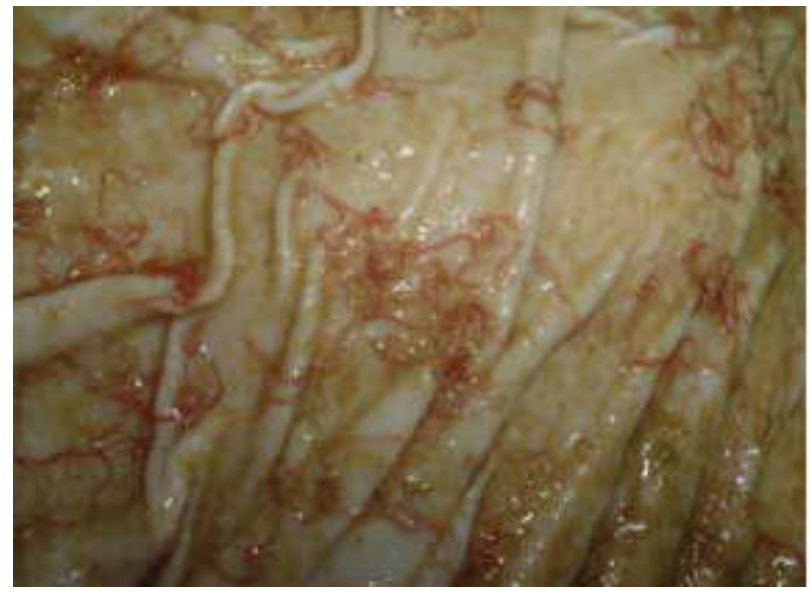

Figure 3 : Vers adultes de Haemonchus contortus sur la paroi interne de l'abomasum (Chrétien, 2011).

\section{SYNTHESE}

Les élevages de bovins Somba connaissent bon nombre de difficultés liées aux facteurs externes tels que la grande prévalence du parasitisme au sein des troupeaux. Ainsi, comme le stipule les travaux de Attindéhou et al. (2012), Salifou et al. (2012), et Chabi China et al. (2013), pour une meilleure viabilité des animaux, la survie et l'extension des troupeaux, le contrôle des facteurs parasitaires d'influence parait très important compte tenu de la forte pression parasitaire qui prévaut en zone tropicale en général, et particulièrement dans l'aire de répartition du bovin Somba. Les moyens classiques de lutte telle que la chimiothérapie vétérinaire ont été mis en œuvre depuis des décennies pour limiter la pression parasitaire sur les animaux. Cette méthode a longtemps connu un grand succès du fait de l'utilisation de molécules à large spectre ayant une action foudroyante sur un grand nombre de parasites. Cette grande efficacité est également relevée par les travaux de Lacroux (2006), et Besier (2007). Mais d'après les travaux de Lumaret et Errouissi (2002), Bishop et Morris (2007), et Chabi China et al. (2014), les éleveurs et les scientifiques se projettent de plus en plus dans l'exploration d'autres moyens de lutte du fait de l'apparition de souches de parasites de plus en plus résistantes aux molécules chimiques, la détérioration de la qualité des produits d'origine animale et bien d'autres limites associées à la chimiothérapie. A cela s'ajoute les difficultés d'accès aux services et aux produits vétérinaires par les éleveurs pauvres, et des contrées reculées comme la plupart des éleveurs de bovins Somba. Selon Brunet, (2008), cette recherche de solutions alternatives à la chimiothérapie peut se faire suivant trois axes à savoir : le tarissement des sources de contamination, l'amélioration de la résistance des animaux, l'élimination des nématodes gastro-intestinaux. Au niveau de ces trois axes des résultats concluants ont été atteints et des méthodes efficaces sont proposées. Cependant, la plupart de ces méthodes, onéreuses, et nécessitant un certain niveau de technicité et de technologie, restent inaccessibles aux élevages traditionnels domestiques africains tels que les élevages de bovins Somba. Comme l'indiquent les travaux de Chabi China et al. (2014), l'élimination des parasites à travers la valorisation des méthodes endogènes reconnues de façon séculaire et basée sur l'ethnomédecine vétérinaire apparait donc comme une alternative pour ces élevages. En effet, en Afrique, un grand nombre d'espèces végétales est déjà utilisé dans les élevages de type traditionnel pour traiter les affections animales (Déléké - Koko et al., 2011; Dibong et al., 2011; Dossou et al., 2012; Fabiyi et al., 2012; Lagnika et al., 2012). Mais ces plantes sont généralement utilisées de façon informelle et sans référentiel médical. Pour aboutir à 
l'usage formel de l'ethnopharmacie en médecine vétérinaire comme alternative crédible à la chimiothérapie dans les élevages de bovins Somba, il y a des conditions préalables à remplir à savoir: faire le répertoire exhaustif des plantes et recettes à bases de plantes utilisées contre les affections courantes du bovin Somba ; faire l'analyse chimique de ces plantes ou organes de plantes utilisés afin de déterminer leur véritable potentiel pharmacologique ainsi que les grands groupes de composés chimiques responsables de cette aptitude thérapeutique ; pour les plantes ayant un fort potentiel pharmacologique, faire des tests biologiques "in vitro" et "in vivo" afin d'établir scientifiquement leur efficacité sur les affections supposées traitées ; pour les plantes dont les essais sont concluants, réaliser des tests de toxicité pour déterminer leur degré de toxicité ; enfin pour les plantes ayant un degré de toxicité acceptable, déterminer les meilleures formes d'utilisation, la dose efficace, la dose utile, la dose économique.

\section{Conclusion}

La race bovine Somba, évolue dans un système d'élevage de type traditionnel caractérisé par des conditions d'élevage difficiles qui plombent l'expression optimale de ses aptitudes productives. Le climat favorable à la prolifération des diverses affections animales dont principalement les parasites crée également un environnement hostile qui affectent davantage les performances productives et limitent le développement et l'expansion de la race. Dans le contrôle du parasitisme au niveau des élevages de bovins Somba l'ethnomédecine se révèle être un atout comme alternative à la médecine vétérinaire moderne peu accessible et de plus en plus décriée. Toutefois, son utilisation, artisanale et informelle, nécessite au préalable une caution scientifique sur la base d'essais expérimentaux concluants.

\section{CONFLIT D'INTERETS}

Les auteurs déclarent qu'il n'existe aucun conflit d'intérêts entre auteurs.

\section{CONTRIBUTIONS DES AUTEURS}

TFCC, ABG et SA ont réalisé l'étude, notamment la collecte de données et la bibliographie. Ils ont également assuré la rédaction du projet d'article. S-YD, SS, LJP et FAA ont contribué à l'étude à travers le suivi des activités, la fourniture de données complémentaires sur l'historique du bétail trypanotolérant en Afrique, et la gestion du risque parasitaire du bovin Somba. Ils également assuré la relecture et la correction du manuscrit.

\section{REFERENCES}

Adanléhoussi A, Bassowa H, Défly A, Djabakou K, Adoméfa K, Kouagou NT. 2003. Les performances de la race taurine Somba en milieu paysan. Tropicultura, 21(3): 135-141.

Adetutu, A, Morgan WA, Corcoran O. 2011. Ethnopharmacological survey and in vitro evaluation of wound-healing plants used in Southwestern Nigeria. Journal of Ethnopharmacology, 137(1): 50- 56.

Ademola IO, Idowu SO. 2006. Anthelmintic activity of Leucaena leucocephala seed extracts on Haemonchus contortus infective larvae. Veterinary Record, 158: 485-486.

Assogba MN, Youssao AKI. 2011. Epidémiologie de la fasciolose à Fasciola gigantica (Cobbold, 1885), de la dicrocoeliose et de la paramphistomose bovines au Bénin. Ann. Méd. Vét., 145: 260-268.

Assogbadjo AE, Amadji G, Glèlè LR, Mama A, Sinsin B, Van Damme P. 2009. Evaluation écologique et ethnobotanique de Jatropha curcas au Bénin. Int. J. Biol. Chem. Sci., 3(5): 1065-1077.

Athanasiadou S, Kyriazakis I, Jackson F, Coop RL. 2000. Effects of short-term exposure to condensed tannins on adult Trichostrongylus colubriformis. Vet. Rec., 146: 728-732.

Athanasiadou S, Kyriazakis I, Jackson F, Coop RL. 2001. Direct anthelmintic effects of condensed tannins towards different gastrointestinal nematodes of sheep: in vitro and in vivo studies. Vet. Parasitol., 99: 205-219. 
Attindéhou S, Salifou S, Biaou CF, Gbati OB, Adamou-N'diaye M, Pangui LJ. 2012. Epidemiology of haemonchosis in sheep and goats in Benin. Journal of Parasitology and Vector Biology, 4(2): 20-24.

Avégan DK, 1984. Etude des aptitudes de la race bovine Somba, importance de son élevage au Togo. Mémoire de fin de cycle pour obtenir le Diplôme d'Ingénieur des sciences appliquées, Institut polytechnique rural de Katibougou, Mali.

Bernes G, Waller PJ, Christensson D. 2000. The effect of birdsfoot trefoil (Lotus corniculatus) and white clover (Trifolium repens) in mixed pasture swards on incoming and established nematode infections in young lambs. Acta Veterinaria Scandinavica, 41: 351-361.

Besier B. 2007. New anthelmintics for livestock: the time is right. Trends in Parasitology, 23(1): 21-24.

Bishop SC, Morris CA. 2007. Genetics of disease resistance in sheep and goats. Small Ruminant Research, 70(1): 48-59.

Brunet S. 2008. Analyse des mécanismes d'action antiparasitaire de plantes riches en substances polyphénoliques sur les nématodes du tube digestif des ruminants. Thèse de l'Institut National Polytechnique de Toulouse.

Chabi China TF, Adénilé A, Doko Allou S, Olounlade PA, HounzangbeAdote MSG, Abiola FA, Salifou S. 2013. Zootechnical study of breeding modes of somba cattle in Benin. Int. J. Biol. Chem. Sci., 7(6): 2193-2201.

Chabi China TF, Olounladé PA, Salifou S. 2014. Ethnobotanical study of endogenous methods used for the treatment of diseases of Somba cattle breed in northen Benin. Journal of Drug Delivery and Therapeutics, 4(3): 1-9.

Chrétien AC. 2011. Cinétique comparée des phénomènes physiopathologiques et de la réponse immune chez des ovins résistants (Martinik Black Belly) ou sensibles (lacaune) au cours d'une primoinfestation par Haemonchus contortus. Thèse pour l'obtention $\mathrm{du}$ grade de
Docteur Vétérinaire, Ecole Nationale Vétérinaire de Toulouse, $157 \mathrm{p}$.

Costa CTC, Bevilaqua CMI, Maciel, MV, Camurca Vasconcelos, ALF, Morais SM, Monteiro MVB, Fraias VM, da Silva MV, Souza MMC. 2006. Anthelmintic activity of Azadirachta indica (Juss) against sheep gastrointestinal nematodes. Vet. Parasitol., 137: 306-310.

De Caluwé E, Halamová K, Van Damme P. 2009. Baobab (Adansonia digitata L.): a review of traditional uses, phytochemistry and pharmacology. In African Natural Plant Products: New Discoveries and Challenges in Chemistry and Quality. Rodolfo H, Simon JE, Ho C-T (Eds). Oxford University Press: USA; 51-84.

Deleke Koko IKE, Djego J, Gbenou J, Hounzangbe-Adote SMG, Sinsin B, 2011. Etude phytochimique des principales plantes galactogènes et emménagogues utilisées dans les terroirs riverains de la Zone cynégétique de la Pendjari. Int. J. Biol. Chem. Sci., 5(2): 618-633.

Dibong SD, Mpondo Mpondo E, Ngoye A, Kwin MF, Betti JL, 2011. Ethnobotanique et phytomédecine des plantes médicinales de Douala. J. Appl. Biosci., 37: 2496-2507.

Domingo AM. 1976. Contribution à l'étude de la population bovine des Etats du Golfe $\mathrm{du}$ Bénin. Thèse de médecine vétérinaire, Ecole Inter-Etats des Sciences et Médecine Vétérinaire de Dakar, Sénégal, 120 p.

Dossa SC. 2000. Preliminary studies on the importance of ticks and tickborne diseases inpurebred Somba in Benin. Project INCO-SOMBA, Report, p. 89

Dossou ME, Houessou GL, Lougbégnon OT, Tenté AHB, Codjia JTC. 2012. Etude ethnobotanique des ressources forestières ligneuses de la forêt marécageuse d'Agonvè et terroirs connexes. Tropicultura, 30(1): 41-48.

Fabiyi M, Goel R, Snowling S, Novak R. 2012. Reduction of VOC Emissions in High Purity Oxygen Activated Sludge 
Wastewater Treatment Process: Toxchem Based Fate \& Emissions Modeling Case Study. AIChE Annual Meeting, Conference Proceedings; Pittsburgh, PA, Oct 2 - Nov 2: American Institute of Chem. Engineers.

Fah L, Klotoé JR, Dougnon V, Koudokpon H, Fanou VBA, Dandjesso C, Loko F. 2013. Étude ethnobotanique des plantes utilisées dans le traitement du diabète chez les femmes enceintes à Cotonou et Abomey-Calavi (Bénin). J. Anim. Plant Sci., 18(1): 2647-2658.

Farougou S, Tassou AW, Txhabode DM, Kpodekon M, Boko C, Youssao AKI. 2007. Tiques et hémoparasites du bétail dans le nord-Bénin. Rév. Méd. Vét., 158(8-9): 463-467.

Hall SJG, Gnaho LK, Meghen C. 1995. Une enquête sur la race bovine Somba au Bénin. Revue Elev. Méd. vét. Pays Trop., 48(1): 77-83.

Hardouin J, Lopez G, Sanaho MN, Fofana BS, Toure SM. 1989. Protection de la race taurine Somba et de son environnement. 1. Etude. 2. Proposition de document de projet, Bénin-Togo, $31 \mathrm{p}$.

Hounzangbé-Adoté S. 2004. Propriétés anthelminthiques de 4 plantes tropicales testées in vitro et in vivo sur les nématodes gastro-intestinaux chez les petits ruminants Djallonké. Thèse de doctorat, Faculté des Sciences et Techniques, Université d'AbomeyCalavi, Benin.

Joshi NR, Mc Laughlin EA, Philips RW. 1957. Types and breeds of African cattle. FAO Agricultural Studies. №37. Rome, Italie, 297 p.

Kahiya C, Mukaratirwa S, Thamsborg SM. 2003. Effects of Acacia nilotica and Acacia karoo diets on Haemonchus contortus infections in goats. Vet. Parasitol., 115: 265-274.

Lacroux C. 2006. Régulation des populations de nématodes gastro-intestinaux dans deux races ovines, INRA 401et Barbados Black Belly. Thèse de doctorat, Institut National Polytechnique de Toulouse, Ecole doctorale Sciences Ecologiques,
Vétérinaires,

Agronomiques et Bioingénieries.

Lagnika L, Murielle HT, Fantodji, Ambaliou Sanni A. 2012. Phytochemical study and antibacterial, antifungal and antioxidant properties of Bridelia ferruginea and Pteleopsis suberosa. IJPSR, 3(7): 21302136.

Lumaret JP, Errouissi F. 2002. Use of anthelmintics in herbivores and evaluation of risks for the non-target fauna of pastures. Vet. Res., 33: 547-562.

Milind P, Gurditta G, 2012. Basketful benefits of papaya. Int. Res. J. Pharm., 7: 6-12.

Moazami-Goudarzi K, Belemsaga DMA, Ceriotti G. 2001. Caractérisation de la race bovine Somba à l'aide de marqueurs moléculaires. Rev. Elev. Méd. Vét. Pays Trop., 54(2): 129-138.

Nguta JM, Mbaria JM, Gakuya DW, Gathumbi PK, Kiama SG. 2010. Antimalarial herbal remedies of Msambweni, Kenya. Journal of Ethnopharmacology, 128: 424-432.

Olounladé PA, Hounzangbé-Adoté MS, Azando EVB , Tamha TB, Brunet S, Moulis C, Fabre N, Fouraste I, Hoste H, Valentin A. 2011. Etude in vitro de l'effet des tanins de Newbouldia laevis et de Zanthoxylum zanthoxylö̈des sur la migration des larves infestantes de Haemonchus contortus. Int. J. Biol. Chem. Sci., 5(4): 1414-1422.

PAMRAD. 2006. Recensement et diagnostic de l'élevage de la race Somba dans la Commune de Boukombé. Rapport provisoire, p. 62.

Prichard R. 2001. Genetic variability following selection of Haemonchus contortus with anthelmintics. Trends Parasito, 17: 445-453.

Salifou S, Daga DF, Attindéhou S. 2013. Prevalence and intensity of nodular oesophagostomosis of in WALL goats in Northern Benin. Journal of Parasitology and Vector Biology, 5(2): 17-19.

Sedogbo - Houenou MD. 1993. Contribution à l'étude des Systèmes d'Elevage Bovin en Afrique de l'Ouest: Analyse de l'expérience du Bénin. Thèse de doctorat 
de Médecine Vétérinaire. Ecole Inter Etat des Sciences et Médecine Vétérinaire (EISMV) Dakar, 153p.

Shaw APM, Host CH. 1987. Trypanotolerant Cattle and Livestock Development in West and Central Africa (Vol.1). The International Supply and Demand for Breeding Stock (Vol. 2). Trypanotolerant Cattle in the National Livestock Economies. FAO Animal Production and Health Papers, Nos 67/1, 67/2. FAO: Rome, Italie.

Varisha A, Husain AS, Javed NK, Poonam A. 2013. Physico-chemical and phytochemical evaluation of Carica Papaya LINN unripe fruits. Int. Res. J. Pharm., 4(8): 101-106.
Waller PJ. 2006. From discovery to development: current industry perspectives for the development of novel methods of helminth control in livestock. Vet. Parasitol., 139(1-3): 1-14.

Youssao Abdou Karim I, Dahouda M, Attakpa EY, Koutinhouin GB, Ahounou GS, Toléba SS, Balogoun BS. 2013. Diversité des systèmes d'élevages de bovins de race bovine Borgou dans la zone soudanienne du Bénin. Int. J. Biol. Chem. Sci., 7(1): 125-146.

Zunjar V, Mohamed D, Trivedi BM, Daniel M. 2011. Pharmacognostic, phytochemical studies on Carica papaya Linn. Leaves. Pharmacognosy Journal, 3 : 5-8. 